\title{
Implementing Continuous Flow over Dense Wireless Sensor Networks
}

\author{
Kamal P. Rezaei, Sina Z. Anaraki, Mehdi Kalantari \\ Department of Electrical and Computer Engineering \\ University of Maryland, College Park \\ \{kamalpr, anaraki, mehkalan\}@umd.edu
}

\begin{abstract}
One of the challenges in routing for dense Wireless Sensor Networks (WSN) is balancing the traffic over different paths. Load-balancing methods inspired by physical phenomena such as electrostatics and optics have been studied in recent years, and the proposed methods are promising in energy-critical routing applications. Such methods model information flow in continuous domain in order to benefit from well-established continuous space optimization methods; however, the existing literature on physics inspired approaches do not address the link between continuous and discrete space models. In this paper, we present a discretization method for linking continuous flow to discrete paths in a dense-WSN. We study a p-norm flow optimization method in continuous domain and show that a fixedpath routing is not sufficient to completely balance traffic load over the network. Our results show that a significant improvement in transport capacity can be achieved by using a dynamic path selection scheme and balancing the traffic over the selected paths.
\end{abstract}

Keywords- dense WSN; routing; load-balancing;

\section{INTRODUCTION}

Rapid advancements in wireless communications and microelectronics have enabled the realization of Wireless Sensor Networks (WSN) which are composed of many low cost, low-power, and small sensor nodes. The small cost of such sensors leads to developing them in large scales and hence enables the concept of dense networks. In such networks, several hundred to several thousand sensors are distributed over a geographical area. Such dense networks have many potential applications in environment monitoring, transportation, surveillance, and agriculture. In a single commodity sensor network, the data produced in individual sensors is collected in one or a few special stations called data sinks (or simply sinks). Nodes forward their data through a multi-hop routing scheme toward one of the sinks.

Designing routing schemes for large number of wireless nodes and careful analysis of the network behavior becomes challenging by using conventional optimization methods based on discrete models. As discrete models consider traffic generation and forwarding only at the locations of the wireless nodes, often the flow analysis becomes computationally prohibitive when the number of nodes grow very large. On the other hand, continuous space models which formulate information flow in the network as a continuous vector field over the whole space seem to be promising, since they can apply continuous domain techniques such as vector calculus, and calculus of variations. While these methods optimize the flow (subject to a flow conservation law) in continuous domain, a real network is implemented by a finite number of nodes. Thus, the optimal flow vector field should be discretized in order to realize a routing protocol. Error will be introduced when a continuous space model is converted to a discrete space model. This is due the fact that in discrete space models the information flow is constrained by sensor locations. In this paper we study the effect of such discretization and show that a statistical multiplexing scheme can significantly reduce the discretization error.

In previous works of the authors, a vector field model was used to model information flow at every point of a network. This idea was first introduced in [1], with a model inspired by electrostatics and using analogy of distribution of an electric field in a dielectric media with information transport in a network with a large number of wireless nodes. In that work, a quadratic cost function is used, and it was shown that solution to the optimization problem is found by solving a set of PDEs analogous to Maxwell's equations in electrostatics.

An extension of the work in [1] to multiple commodity flow networks was given in [2]. Further generalization to convex optimization of network flow was introduced in [3]. In special cases, the optimization function can be used to minimize the $p$-norm of information flow vector field subject to the basic flow constraints (i.e., flow conservation and boundary constraints). This method is called $p-$ norm flow optimization. It is shown that theoretically, $p-$ norm flow optimization in the special case of $p$ being considerably large exploits the most possible capacity for load balancing of traffic especially around sinks. It should be emphasized that as battery power in WSNs is limited and the lifetime of network is determined by first bottleneck nodes batteries being depleted, balancing traffic load around sinks increases the overall lifetime of the network [5].

While we proved the maximal load balancing property of the $p$-norm scheme in continuous domain, it became necessary to develop a method for approximating flow field in a discrete space WSN. It is obvious that nodes in some regions of the network and especially in the sparse areas can not forward their packets exactly in the direction that flow vector filed dictates, and some discretization errors are introduced. These errors are of the same nature of analog to digital signal

This work was partially supported by NSF under grant CCF-0729129 and grant 0931957 
conversion at low precisions. The routing scheme approximating the flow vector field may deviate from its balanced state.

In this paper, we present a scheme to considerably limit discretization errors by dynamically switching paths to achieve asymptotic time-averaged discrete flow aligned with continuous field flow at the place of each node. We show that at most only two paths are contributing to render continuous like flow at the node.

The rest of the paper is organized as follows. In section II, we give a background on a quasi-balanced electrostaticsinspired routing scheme followed by more sophisticated $p$ - norm scheme for dense WSNs. In section III, we illustrate the nature of flow discretization error in such dense WSNs. Next section is allocated to describing our approach to discretization of continuous field flow. In section $\mathrm{V}$, we provide simulation results showing load-balancing property of the network routed with $p-$ norm continuous flow field. Section VI concludes the paper.

\section{BACKGROUND}

In order to model flow of information in a network, we define a vector quantity and call it information flow vector field. The network is located in a closed, connected, and bounded set $A \in R^{2}$. The information sources are distributed with a spatial density function $\mu(z) \in A$, which means that at location $z=(x, y) \in A-\left\{\left(x_{0}, y_{0}\right)\right\}, \quad \rho(z) \mathrm{bps} / \mathrm{m}^{2} \quad$ of information is generated. A sink located at $z_{0}=\left(x_{0}, y_{0}\right)$ absorbs all this information, so $\rho\left(z_{0}\right)$ has a minus Dirac delta function form. Let $\boldsymbol{D}(z)=\left(D_{x}, D_{y}\right)$ denote the vector field whose direction represents the direction of flow of information at point $z$, and its magnitude represents the information rate passing per unit length of a line segment perpendicular to the direction of $\boldsymbol{D}(z)$. In other words, if we consider a line segment with a small length $\Delta l$ at $z$ and perpendicular to the direction of $\boldsymbol{D}(z)$, the information crosses that line segment with rate $D(z) \Delta l$. The above definition of $\boldsymbol{D}(z)$ implies that for a closed contour $C \in A$ we have:

$$
\oint_{C} \boldsymbol{D}(z) \cdot \boldsymbol{d n}=\int_{S(C)} \rho(z) \cdot d x d y
$$

or:

$$
\nabla \cdot \boldsymbol{D}(z)=\rho(z)
$$

in which $\boldsymbol{d n}$ is a differential vector normal to the contour at each point of its boundary and pointing to the outside of the contour, the dot represents the inner product of vectors in twodimensional space, and $S(C)$ is the area surrounded by the closed contour $C$. This equation states the flow conservation law in dense networks; however, it does not define the vector field $\boldsymbol{D}$ uniquely. This opens the room for finding a flow vector field that minimizes a cost function. In [1,2] we introduced a quadratic cost function of the information flow vector field $\boldsymbol{D}$ in order to find a unique solution:

$$
\begin{array}{cl}
\text { Minimize } & J(D)=\int_{A}|\boldsymbol{D}(z)|^{2} d x d y \\
\text { s.t. } & \nabla . \boldsymbol{D}(z)=\rho(z) \\
& D_{n}(z)=0 z \in \partial A
\end{array}
$$

The constraints of the above optimization problem ensure that first, how flow is related to sources and sinks and second, no flow leaks out of the network boundary $\partial A$. The above form of cost function results in an optimal flow vector field with a spatial spreading of the communication load over the geographic area of the network. To some extent, it balances the communication load in such a way that it avoids having large traffic load somewhere in the network while the resources are underutilized somewhere else.

As the quadratic cost function does not achieve maximum spatial spreading of the traffic over the network, we proposed the following general form of optimization problem:

$$
\begin{array}{cl}
\text { Minimize } & J(D)=\int_{A}|\boldsymbol{D}(z)|^{p} d x d y \\
\text { s.t. } & \nabla . \boldsymbol{D}(z)=\rho(z) \\
& D_{n}(z)=0 z \in \partial A
\end{array}
$$

in which $p>1$ is a real number. In [3] it is shown that increasing the value of $p$ causes the optimization problem to increase the amount of spatial spreading in the network. This is equivalent to a highly load-balanced flow vector field in the network.

\section{PROBLEM DESCRIPTION}

Load-balancing methods like electrostatics-based flow optimization provide the continuous domain information flow vectors; however, they should be implemented with discrete paths between nodes in a practical dense WSN. In general, it is desirable to minimize the difference between flow field $\boldsymbol{D}(x, y)$ and implemented flow using the network nodes. For this purpose, we first describe the nature of discretization error in a dense WSN.

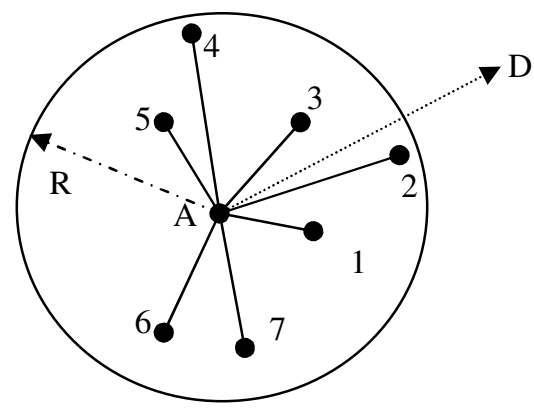

Figure 1. node A with 7 neighbors inside its communication range

In a random node placement with uniform surface node density, $\sigma$ and node communication range, $R$, we have $\sigma \pi R^{2}$ nodes on average in communication range of each node. Therefore, there are $\sigma \pi R^{2}-1$ neighbors for a node denoted by $A$. We assume that $\boldsymbol{D}\left(x_{A}, y_{A}\right)$ is flow vector at the location of node $A$. Also, we define $\boldsymbol{V}_{\boldsymbol{i}}$ as the vector along path $A i$, connecting $A$ to its neighbor node $i$. Node $A$ can forward to any of its neighbors, so for example in figure 1 there are 7 vectors $\boldsymbol{V}_{\mathbf{1}}, \boldsymbol{V}_{\mathbf{2}}, \ldots, \boldsymbol{V}_{\mathbf{7}}$. No matter which of the neighbors is chosen for forwarding the traffic, there is a deviation between flow $\boldsymbol{D}$ determined in a load-balanced solution and its instantaneous direction $\boldsymbol{V}_{\boldsymbol{i}}$ imposed by discrete nature of the

This work was partially supported by NSF under grant CCF-0729129 and grant 0931957 
network. In a simplistic treatment of the problem, it seems that as we increase $\sigma$ or $R$ node $A$ will have more choices of the neighbors to forward its traffic hence the deviation angles between $\boldsymbol{D}$ and $\boldsymbol{V}_{\boldsymbol{i}}$ tend to be smaller; however, the following discussion shows that this intuition is not true and in general it is not helpful to use too many neighbor nodes surrounding node $A$.

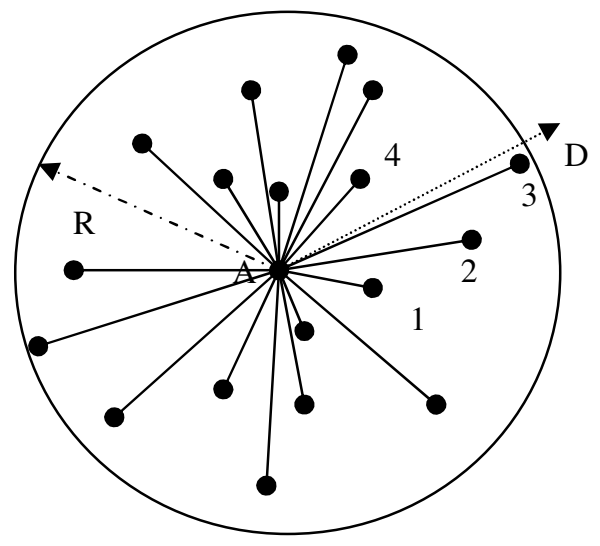

Figure 2. network with larger $\rho$ or $R$

As shown in figure 2, by increasing the number of neighbors, the distance between a node and its neighbors tends to have a larger variance. Then, it is likely that at least one of the paths closely siding $\boldsymbol{D}$ is far from it. By choosing a long path, similar to $A 3$ shown in figure 2, although the direction along which the information is forwarded gets close to the orientation of $\boldsymbol{D}$, we may jump to another part of the network in which direction and magnitude of $\boldsymbol{D}$ may have changed considerably. In other words, it is important to limit hops to a few nodes surrounding $A$ where $\boldsymbol{D}$ is roughly constant over all such nodes in the vicinity of $A$.

\section{FROM CONTINUOUS FLOWS TO DISCRETE PATHS}

As discussed in last section, even in highly dense networks with large $\sigma R$, it is likely that we will not find paths that closely match the direction of $\boldsymbol{D}\left(x_{A}, y_{A}\right)$ at location of any node like $A$. Based on this fact, we devise a statistical path switching scheme to give a time-averaged $\overline{\boldsymbol{D}}$ asymptotically following the direction of $\boldsymbol{D}\left(x_{A}, y_{A}\right)$ :

$$
\overline{\boldsymbol{D}}=\sum_{i=1}^{N_{N B}} p_{i} \widehat{\boldsymbol{V}}_{\boldsymbol{i}}\left|\boldsymbol{D}\left(x_{A}, y_{A}\right)\right|
$$

where $N_{N B}$ is number of the neighbors, $p_{i}$ is probability of selecting path $A i$ and $\widehat{\boldsymbol{V}}_{\boldsymbol{i}}$ is unit vector along this path. As we will show later, it is not necessary to have contributions of all $\widehat{\boldsymbol{V}}_{\boldsymbol{i}}$ 's and in fact we only need to consider two paths closely $\operatorname{siding} \boldsymbol{D}\left(x_{A}, y_{A}\right)$. Angles between $\boldsymbol{D}\left(x_{A}, y_{A}\right)$ and these two paths should be less than $90^{\circ}$ and we get faster convergence toward $\boldsymbol{D}\left(x_{A}, y_{A}\right)$ as these angles are smaller. It is not always possible to have this overall information flow vector exactly coinciding $\boldsymbol{D}$, however, under sufficient densities in relation to communication range, $R$, we can make time-averaged direction of flow to be aligned with $\boldsymbol{D}$.

The problem is how to calculate probabilities, $p_{i}$, for selecting path $A i$, so that expected flow direction is $\boldsymbol{D}$ according to (1). We present an inductive method here to prove our claim that only two nearest paths on both sides of $\boldsymbol{D}$ are enough in our summation to make the best local approximation to $\boldsymbol{D}$ at node's place. First, we postulate that packets are forwarded toward paths which have positive contribution for $\boldsymbol{D}$. In other words, as figure 3 shows, forwarding path should be at the same side of line D', drawn perpendicular to $\boldsymbol{D}$ in node A, where $\boldsymbol{D}$ lies.

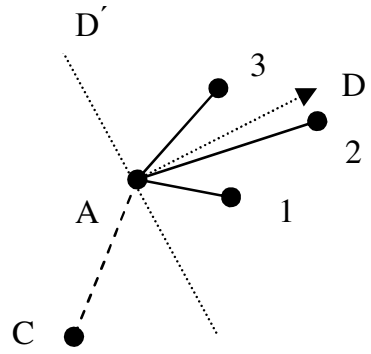

Figure 3. node A with 3 forwarding paths and 1 retrograde path

Then, $\boldsymbol{V}_{\boldsymbol{i}}$ has a positive projection alongside $\boldsymbol{D}$. Implications for such postulate come from the fact that there should be a zero probability for loops. Supposed as figure 3 shows retrograde path like $A C$ is chosen from $A$. It is obvious that now path $C A$ can be chosen from $C$, and so we get a non-zero probability for traversing loop $A C-C A$. We define half-plane $\boldsymbol{D}$ as the region of plane where vector $\boldsymbol{D}$ (drawn from $\mathrm{A}$ ) lies.

For the one path case, where $A$ can only forward its packet to another node in half-plane $\boldsymbol{D}$, we have to tolerate the direction error made by angle between path and $\boldsymbol{D}$. Now, we consider two paths case, where paths lay on both sides of $\boldsymbol{D}$, but certainly in half-plane $\boldsymbol{D}$. Figure 4 shows such case with paths $\widehat{\boldsymbol{V}_{\mathbf{1}}}$ and $\widehat{\boldsymbol{V}_{\mathbf{2}}}$ making angles $\theta_{1}$ and $\theta_{2}$ respectively.

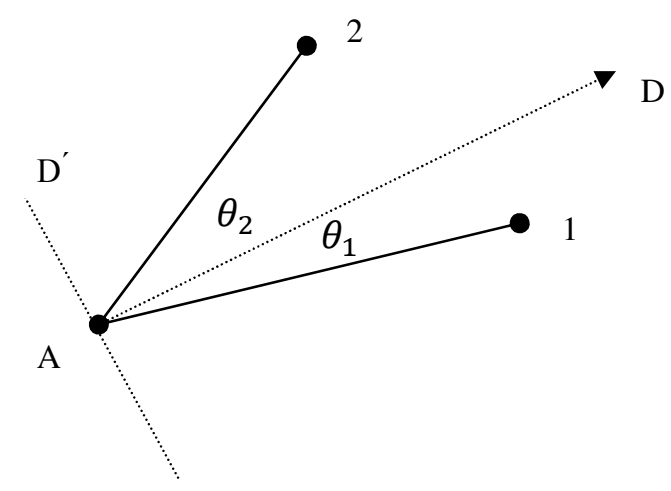

Figure 4. node A with 2 forwarding paths on half-plane $D$ 
We should determine weighing factors $p_{i}$ 's so that:

$$
\begin{array}{r}
\widehat{\mathbf{D}}=\mathrm{p}_{1} \widehat{\boldsymbol{V}_{1}}+\mathrm{p}_{2} \widehat{\boldsymbol{V}_{2}} \\
\mathrm{p}_{1}+\mathrm{p}_{2}=1
\end{array}
$$

It means that component of $\widehat{\boldsymbol{V}}_{\boldsymbol{i}}(i=1,2)$ normal to $\boldsymbol{D}$ should cancel out each other. Thus, we must have:

$$
\left|\widehat{\boldsymbol{V}_{1}}\right| \mathrm{p}_{1} \cos \left(\frac{\pi}{2}-\theta_{1}\right)=\left|\widehat{\boldsymbol{V}_{\mathbf{2}}}\right| \mathrm{p}_{2} \cos \left(\frac{\pi}{2}-\theta_{2}\right)
$$

or:

$$
\mathrm{p}_{1} \sin \left(\theta_{1}\right)=\mathrm{p}_{2} \sin \left(\theta_{2}\right)
$$

We can simply find $\mathrm{p}_{1}$ and $\mathrm{p}_{2}$ according to the angles of their paths with $\boldsymbol{D}$ as followings:

$$
\left\{\begin{array}{l}
\mathrm{p}_{1}=\frac{\sin \left(\theta_{2}\right)}{\sin \left(\theta_{1}\right)+\sin \left(\theta_{2}\right)} \\
\mathrm{p}_{2}=\frac{\sin \left(\theta_{1}\right)}{\sin \left(\theta_{1}\right)+\sin \left(\theta_{2}\right)}
\end{array}\right.
$$

This result shows that $p_{i}$ gets larger as angle $\theta_{i}$ becomes smaller. In the special case of $\theta_{i}=0$, we get $p_{i}=1$, and so the coinciding path is selected always as was expected. Also case $\theta_{1}=\theta_{2}$ gives us another intuitive result that in this situation paths are equally likely to be selected. If both paths are in one side of $\boldsymbol{D}$, it is obvious that the farther path, i.e. the one with larger $\theta_{\mathrm{i}}$, should not be traversed at all, as it increases the direction error already built by nearer path.

Now, we consider the case where there are three paths in half-plane $\boldsymbol{D}$ (figure 5).

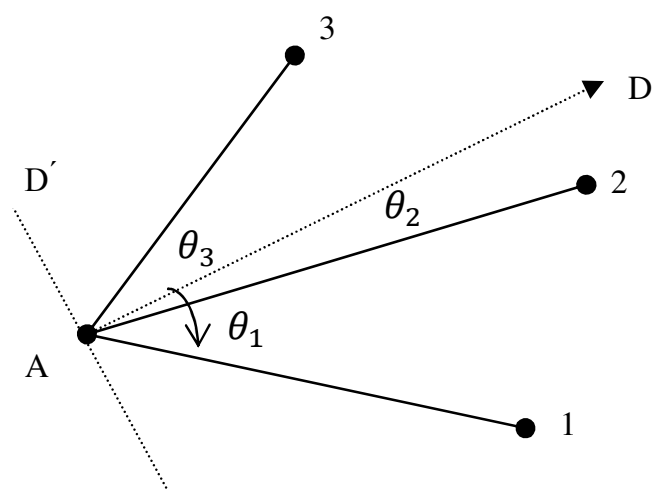

Figure 5: node A with 3 forwarding paths

Here, we can write following two constraints for $p_{1}$, $p_{2}$ and $p_{3}$, probabilities of traversing three paths from node $A$ to have an overall flow vector coinciding $\boldsymbol{D}$ :

$$
\left\{\begin{array}{c}
\left|\widehat{\boldsymbol{V}_{1}}\right| p_{1} \cos \left(\frac{\pi}{2}-\theta_{1}\right)+\left|\widehat{\boldsymbol{V}_{2}}\right| p_{2} \cos \left(\frac{\pi}{2}-\theta_{2}\right) \\
-\left|\widehat{\boldsymbol{V}_{3}}\right| p_{3} \cos \left(\frac{\pi}{2}-\theta_{3}\right)=0 \\
p_{1}+p_{2}+p_{3}=1
\end{array}\right.
$$

Therefore, we have one degree of freedom to add another constraint for specifying three probabilities. We use our freedom to make the magnitude of effective flow vector at $A$ as large as possible. In other words, for $N$ consecutive packets arriving at $A$ and forwarded via one of the paths, we should try to keep the error between $\boldsymbol{D}$ and the effective flow minimum. If $n_{i}$ packets are sent via path, then $n_{i}$ should be determined so that component of flow along $\boldsymbol{D}$ attains its maximum:

$$
\begin{gathered}
\max \left\{\left|\widehat{\boldsymbol{V}_{\mathbf{1}}}\right| n_{1} \cos \left(\theta_{1}\right)+\left|\widehat{\boldsymbol{V}_{\mathbf{2}}}\right| n_{2} \cos \left(\theta_{2}\right)\right. \\
\left.+\left|\widehat{\boldsymbol{V}_{\mathbf{3}}}\right| n_{3} \cos \left(\theta_{3}\right)\right\}
\end{gathered}
$$

Which is rewritten as:

$$
\begin{aligned}
& \max N \times\left\{\left|\widehat{\boldsymbol{V}_{\mathbf{1}}}\right| \frac{n_{1}}{N} \cos \left(\theta_{1}\right)+\left|\widehat{\boldsymbol{V}_{2}}\right| \frac{n_{2}}{N} \cos \left(\theta_{2}\right)+\right. \\
& \left.\left|\widehat{V_{3}}\right| \frac{n_{3}}{N} \cos \left(\theta_{3}\right)\right\}
\end{aligned}
$$

In the limit when $N \rightarrow \infty, \frac{n_{i}}{N}$ is replaced with probability $p_{i}$ :

$$
\begin{gathered}
\max \\
\left.\left|\widehat{\boldsymbol{V}_{3}}\right| p_{3} \cos \left(\theta_{3}\right)\right\}
\end{gathered}
$$

We have two constraints for maximizing (4). Omitting magnitude of unit vectors from (4) and (3), we get following linear optimization problem:

$$
\left\{\begin{array}{c}
\max \left\{p_{1} \cos \left(\theta_{1}\right)+p_{2} \cos \left(\theta_{2}\right)+p_{3} \cos \left(\theta_{3}\right)\right\} \\
p_{1} \sin \left(\theta_{1}\right)+p_{2} \sin \left(\theta_{2}\right)-p_{3} \sin \left(\theta_{3}\right)=0 \\
p_{1}+p_{2}+p_{3}=1
\end{array}\right.
$$

By first omitting $p_{3}$ :

$$
\left\{\begin{array}{c}
\max \left\{\begin{array}{c}
p_{1}\left(\cos \left(\theta_{1}\right)-\cos \left(\theta_{3}\right)\right)+p_{2}\left(\cos \left(\theta_{2}\right)-\cos \left(\theta_{3}\right)\right) \\
+\cos \left(\theta_{3}\right)
\end{array}\right\} \\
p_{1}\left(\sin \left(\theta_{1}\right)+\sin \left(\theta_{3}\right)\right)+p_{2}\left(\sin \left(\theta_{2}\right)+\sin \left(\theta_{3}\right)\right) \\
-\sin \left(\theta_{3}\right)=0
\end{array}\right.
$$

and then $p_{2}$, we get:

$$
\begin{aligned}
\max \left\{p _ { 1 } \left(\cos \left(\theta_{1}\right)\right.\right. & \left.-\cos \left(\theta_{3}\right)\right) \\
& -p_{1} \frac{\sin \left(\theta_{1}\right)+\sin \left(\theta_{3}\right)}{\sin \left(\theta_{2}\right)+\sin \left(\theta_{3}\right)}\left(\cos \left(\theta_{2}\right)\right. \\
& \left.-\cos \left(\theta_{3}\right)\right)+\frac{\sin \left(\theta_{3}\right)}{\sin \left(\theta_{2}\right)+\sin \left(\theta_{3}\right)} \\
& \left.+\cos \left(\theta_{3}\right)\right\}
\end{aligned}
$$

Simplifying above trigonometric relation and ignoring constant part in calculating max we deduce following: 


$$
\begin{aligned}
\max \left\{p _ { 1 } \left(\operatorname { s i n } \left(\theta_{2}\right.\right.\right. & \left.\left.\left.-\theta_{1}\right)+\sin \left(\theta_{3}+\theta_{1}\right)-\sin \left(\theta_{3}+\theta_{2}\right)\right)\right\} \\
& =\max \left\{p_{1} A\left(\theta_{1}, \theta_{2}, \theta_{3}\right)\right\}
\end{aligned}
$$

We show that coefficient of $p_{1}$ in above relation is always negative. $A\left(\theta_{1}, \theta_{2}, \theta_{3}\right)$ is re-arranged as:

$$
\begin{aligned}
A\left(\theta_{1}, \theta_{2}, \theta_{3}\right)= & \sin \left(\left(\theta_{3}+\theta_{2}\right)+\left(\theta_{1}-\theta_{2}\right)\right)-\sin \left(\theta_{3}+\theta_{2}\right) \\
& -\sin \left(\theta_{1}-\theta_{2}\right)
\end{aligned}
$$

This is of the form $\sin (\alpha+\beta)-\sin (\alpha)-\sin (\beta)$ and it simply can be checked that for $0<\alpha, \beta \leq \pi$ this expression is less than or equal to zero:

$$
\begin{aligned}
\sin (\alpha+\beta)-\sin & (\alpha) \\
& -\sin (\beta) \\
& =\sin (\alpha)(\cos (\beta)-1) \\
& +\sin (\beta)(\cos (\alpha)-1) \leq 0
\end{aligned}
$$

Therefore, $A\left(\theta_{1}, \theta_{2}, \theta_{3}\right)$ is always nonpositive, and it leads us to choose a least value for $p_{1}$ to maximize (5). Thus, we should set it to zero meaning that path 1 should never be selected. If path 1 is on one side of $\boldsymbol{D}$ and paths 2 and 3 are on the other side of $\boldsymbol{D}$, then second and third terms in relation (3-1) switch signs and we can write $p_{3}$ in the same form of $p_{1}$ as above. It follows that $p_{3}$ should be zero instead of $p_{1}$ in this case. The conclusion is that from three paths it is only necessary to forward packets via two paths closely siding $\boldsymbol{D}$, and we have already calculated probabilities for these two paths. If 3 paths are on one side of $\boldsymbol{D}$, then it is obvious that always the closest path to $\boldsymbol{D}$ should be traversed as others have smaller components along $\boldsymbol{D}$.

Now, we study the case where we have 4 or higher number of paths in half-plane $\boldsymbol{D}$. We can always select 3 adjacent paths with two of them on one side and third one on the other side of $\boldsymbol{D}$ or all of them on one side of $\boldsymbol{D}$. We argue that supposed one of paths in this set is going to be chosen, then optimizing conditional probabilities $p_{C 1}, p_{C 2}$ and $p_{C 3}$ we end up with two paths on both sides or one path on one side of $\boldsymbol{D}$. Putting remained path(s) into another set including the path 4, and using the same argument leaves us with one or two paths closely siding $\boldsymbol{D}$. In a general case where we have $m$ paths in half-plane $\boldsymbol{D}$, with two paths $i$ and $i+1$ closely siding $\boldsymbol{D}$, the same approach leads us to reject first path $i-1$, then path $i+2$, and so on and so forth. Finally, we observe that probabilities of choosing all paths except $i$ and $i+1$ vanish. This important result allows time-averaged discrete domain flow to asymptotically follow $\boldsymbol{D}$ in the continuous domain.

\section{SIMULATION RESUlTS}

In this section, we present simulations and their results for verification of load-balancing behavior of the continuous flow $p$-norm scheme. As it was stated earlier, the most critical part of a dense WSN requiring intensive care regarding energy consumption balancing is the area surrounding sink. Therefore, simulation experiments designed to address this part of the network.
The network we studied consists of 2500 relay nodes spread over a unit square. A sink was placed at coordination $(0.5,0.5)$. Two sets, each including ten sources, have been put at far left and far right sides of the square. Traffic is only generated at these sources and relay nodes just forward it toward sink. Communication range, $R$, has been set to 0.035 .

First part of the simulation code assigns positive charges to 20 sources and negative charge equal in absolute value to the source. In fact, charge densities are allocated to sources and sink to avoid singularity problem arising from point like sources and sink. Based on mentioned geometry and charge distributions, this part of code, first computes flow $\boldsymbol{D}_{2}$ according to quadratic cost function and then refines it through multiple iterations to get a 8 -norm flow $\boldsymbol{D}$. Figure 6 shows diagram of a network with 8-norm flow vectors depicting directions where packets should flow while moving from a source toward sink. It is obvious that this flow field doesn't depend on distribution of relay nodes.

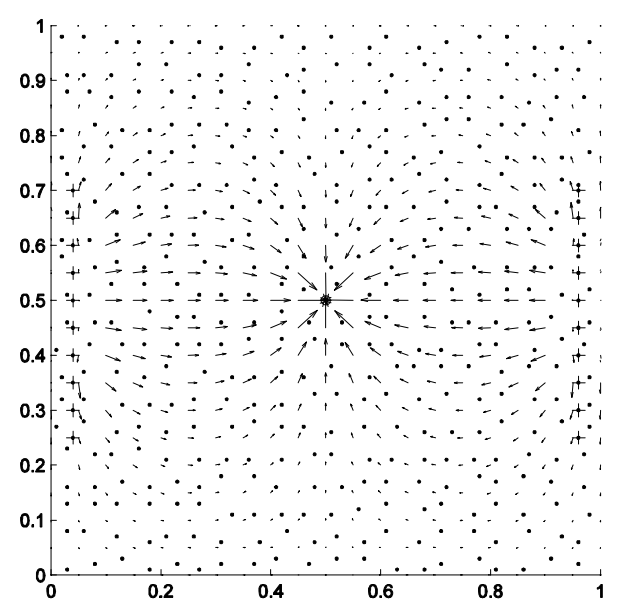

Figure 6: 8-norm flow field

As can be seen in figure 6 , flow shows its balancing behavior in the area encompassing sink. Thus, we expect that this feature be transferred to the discrete information flow between nodes in this area of the random network. To analyze traffic over this area, we introduce the notion of 'Hopping Level' of nodes around a sink. We say that node $\mathrm{N}$ belongs to Hopping Level $i$ of sink $\mathrm{S}$, written as $H L_{i}$, if forwarding packets from $\mathrm{N}$ to $\mathrm{S}$ needs at least $i$ paths (hops). It is obvious that in a load-balanced routing scheme, traffic passed through all nodes in the same hopping level should be equal.

Here, we analyze traffic load of nodes up to level 4 . We assume that traffic generated by sources have been received equally over all nodes in $H L_{4}$. Thus, it is assumed that relay nodes of $H L_{4}$, act as virtual sources re-generating traffic evenly distributed over $H L_{4}$ nodes. The last part of the simulation directs this traffic according to keeping $\overline{\boldsymbol{D}}$ at each node closely matching continuous flow $\boldsymbol{D}$ based on the 


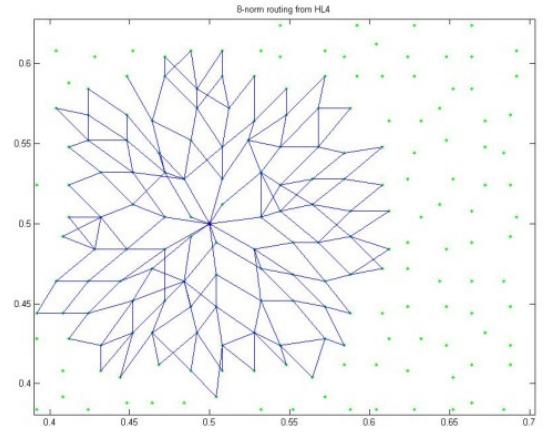

(a)

Figure 7: a) diagram shows vicinity of sink up to Hopping Level 4 for a random network. Paths shown have been used at least once for forwarding packets toward sink. b) The blue histogram shows forwarded traffic by each of 130 nodes in HL1 to HL4 of the network in (a) according to 8-norm routing. Red plot shows idealistic load-balanced traffic for each Hopping Level starting from HL1.

approach developed in last section. A typical traffic of 100 is generated by $H L_{4}$ nodes and diffused toward sink by passing through $H L_{3}, H L_{2}$ and finally $H L_{1}$. The idealistic balanced traffic per node transferred in $H L_{i}(1<i<3)$ is calculated by this formula:

$$
\operatorname{TrfHL}(i)=\operatorname{Trf} H L(4) \times \frac{N H L(4)}{N H L(i)}
$$

Where $N H L(i)$ means number of the relay nodes in $H L_{i}$ and $\operatorname{TrfHL}(i)$ function gives traffic per node of $H L_{i}$. Figure 7 depicts both the routing paths traversed in first 4 hopping levels of a typical network, and the traffic passed through each node in this area. Since simulated traffic is considerably high, all potential paths have been traversed at least once in this network, and increasing traffic to for example 1000 doesn't give us new paths.

The traffic plot have been shown over a step-wise plot depicting idealistic balanced traffic of nodes in $H L(i)$. It simply can be seen that first, critical nodes of $H L 1$ doesn't peak on some nodes as in shortest path routing schemes and second, peaks of $H L(i)$ are smaller than peaks in its inner layer.

\section{CONCLUSION}

In this paper, we presented a scheme to discretize the optimized continuous domain routing flow field in dense WSNs. The discretization is very important as more and more robust continuous space flow optimization methods are developed in comparison to discrete space flow optimizations. These models formulate information flow in the network as a continuous vector field over the whole space and apply wellestablished continuous domain techniques such as vector calculus.

We discussed that discretization errors introduced with fixed routes in a network may not fully transfer the optimization property of continuous flow to the discrete

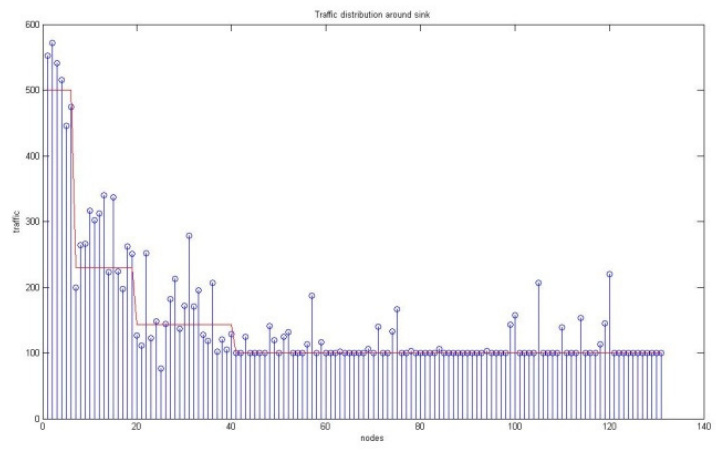

(b) network. Considering this fact, we introduced our scheme based on dynamically switching special paths to achieve asymptotic time-averaged discrete flow in coincidence with continuous flow vector field. We showed that at most only two paths are contributing to render continuous like flow at each node, and using other paths only increases flow discretization error.

Our simulation results verified this proposition by showing how discrete flow in a typical network is inheriting the balanced property of a $p-$ norm continuous space optimization. Based on our work here, we postulate that dynamical switching should be inherent to all routing schemes in dense WSNs. In other words, load-balancing feature of a routing emerges over time, and no scheme can claim instantaneous balancing for a random network. We leave it to the future extensions of this work to prove it.

\section{REFERENCES}

[1] M. Kalantari and M. Shayman, "Routing in wireless ad hoc networks by analogy to electrostatic theory," in Proceedings of IEEE International Communications Conference (ICC-04), June 2004.

[2] M. Kalantari and M. Shayman, "Routing in multi-commodity sensor networks based on partial differential equations," Princeton University, March 2006.

[3] M. Kalantari, M. Haghpanahi and M. Shayman, "A p-norm flow optimization problem in dense wireless sensor networks," in Proceedings of IEEE Infcom 2008.

[4] J. Karaki and A. Kamal, "Routing techniques in wireless sensor networks: a survey," IEEE Wireless Communications Journal, December 2004

[5] S. Toumpis, R. Catanuto and G. Morabito, "Optical / optimal routing in massively dense wireless networks," in Proceedings of IEEE Infcom 2007.

[6] R. Kacimi, R. Dhaou, A. Beylot, "Load-balancing strategies for lifetime maximizing in wireless sensor networks," IEEE International Conference on Communications (ICC), 2010

[7] Y. Chen and Q. Zhao, "On the lifetime of Wireless sensor networks," IEEE Communications Letter, Vol. 9, No 11, pp. 976-978.

[8] E. Hyytia and J. Virtamo, "On traffic load distribution and load balancing in dense wireless multi hop networks," EURASIP Journal on Wireless Communications and Networking, 2007. 